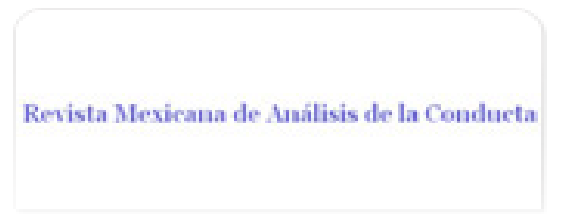

Revista Mexicana de Análisis de la Conducta ISSN: 0185-4534

editora@rmac-mx.org

Sociedad Mexicana de Análisis de la Conducta México

Gómez-Romero, José Guadalupe; González-López, María R. A.; García-Saldívar, Norma; Arriaga, J.

C. Pedro; Cruz-Morales, Sara Eugenia

Disociación de los efectos amnésicos y ansiolíticos en el laberinto elevado en T

Revista Mexicana de Análisis de la Conducta, vol. 32, núm. 2, diciembre, 2006, pp. 271-290

Sociedad Mexicana de Análisis de la Conducta

Guadalajara, México

Disponible en: http://www.redalyc.org/articulo.oa?id=59332211

- Cómo citar el artículo

- Número completo

- Más información del artículo

Página de la revista en redalyc.org

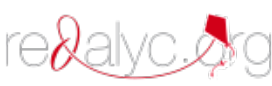

Sistema de Información Científica

Red de Revistas Científicas de América Latina, el Caribe, España y Portugal Proyecto académico sin fines de lucro, desarrollado bajo la iniciativa de acceso abierto 


\title{
DISOCIACIÓN DE LOS EFECTOS AMNÉSICOS Y ANSIOLÍTICOS EN EL LABERINTO ELEVADO EN T
}

\author{
DISSOCIATION OF ANXIOLYTIC AND AMNESIC EFFECTS \\ IN THE ELEVATED T-MAZE. \\ JOSÉ GUADALUPE GÓMEZ-ROMERO, \\ MARÍA R. A. GONZÁLEZ-LÓPEZ., NORMA GARCÍA-SALDÍVAR, \\ J. C. PEDRO ARRIAGA Y SARA EUGENIA CRUZ-MORALES* \\ PSICOFARMACOLOGÍA, FES-IZTACALA, UNAM, MÉXICO.
}

\begin{abstract}
RESUMEN
La administración de benzodiazepinas produce efectos ansiolíticos y amnésicos. Los ansiolíticos no benzodiazepínicos, como la buspirona (BU), parecen tener pocos efectos amnésicos, pero los resultados obtenidos varían con los procedimientos usados para evaluar esos efectos. El laberinto elevado en T (LET) es un procedimiento que permite evaluar al mismo tiempo el efecto amnésico y el ansiolítico. En el presente estudio comparamos los efectos de la administración de escopolamina (E), un antagonista colinérgico clásico con efectos amnésicos, y de dos ansiolíticos, diazepam (DZ) y buspirona sobre la ejecución en el LET. Para ello, ratas Wistar machos (200-250 g) fueron evaluadas en el LET después de uno de los siguientes tratamientos: E (0.5, $1,3 \mathrm{mg} / \mathrm{kg}), \mathrm{DZ}(2,5 \mathrm{mg} / \mathrm{kg}), \mathrm{BU}(2,5 \mathrm{mg} / \mathrm{kg})$ o salina (SAL). El día de entrenamiento cada sujeto fue inyectado y puesto en el brazo cerrado y se midió el tiempo de permanencia en este brazo (evitación) (EV), lo cual fue seguido por otros dos ensayos de EV. A continuación, cada sujeto fue puesto en el brazo abierto y se midió el tiempo en que el sujeto tardó en volver al brazo cerrado (latencia de escape) (ES). En la prueba de retención las latencias de EV y de ES fueron registradas a las 24 h sin la administración de los fárma-
\end{abstract}

* Dra. Sara E. Cruz Morales. Apdo. Postal 314, Tlalnepantla, Edo. de México, 54090, México. Apoyado PAPIIT-IN301102, DGAPA, UNAM, México. 
cos. Se observó un deterioro importante en las latencias de evitación en la adquisición y retención en los grupos tratados con $\mathrm{E}$ en comparación con los que recibieron salina; no se observaron cambios en las latencias de escape. Efectos similares, pero en menor grado fueron observados en las latencias de evitación y retención en los grupos tratados con DZ; se observó un aumento en las latencias de escape sugiriendo un efecto ansiolítico. La BU no tuvo efectos en las latencias de evitación en la adquisición y en la retención; en los animales tratados con la mayor dosis de BU se observó un aumento en las latencias de escape en el primer día, por lo que se sugiere que el fármaco tuvo un efecto ansiolítico. A partir de los resultados del estudio sugerimos que la $E$ induce efectos amnésicos pero no ansiolíticos, mientras que el DZ y la BU tienen efectos ansiolíticos; pero sólo el DZ induce amnesia anterógrada, y que estos efectos pueden ser disociados usando el LET.

Palabras clave: memoria, ansiedad, laberinto elevado en T, escopolamina, diazepam, buspirona.

\begin{abstract}
Benzodiazepines administration produces amnesic and anxiolytic effects. The non-benzodiazepine anxiolytics, like buspirone (BU), seems to have low amnesic effects, but the results obtained vary with the procedures used to evaluate these effects. The elevated T-maze (ETM) is a procedure that allows evaluating both, the amnesic and anxiolytic effects. In the present study, we examined the effect of the administration of a cholinergic antagonist, scopolamine (SC), a classical amnesic agent, and two anxiolytics, diazepam (DZ) and $\mathrm{BU}$ in the ETM. Male Wistar rats $(200-250 \mathrm{~g})$ were tested in the ETM during $5 \mathrm{~min}$ after one of the following treatments: SC $(0.5,1,3 \mathrm{mg} / \mathrm{kg}$, DZ $(2,5 \mathrm{mg} / \mathrm{kg}), \mathrm{BU}(2,5 \mathrm{mg} / \mathrm{kg})$ or saline $(\mathrm{SAL})$. In training day each subject was injected and placed in the enclosed arm and the time remaining in this arm was registered; two subsequent avoidance trials (AV) were given. After avoidance trials, each subject was placed in the open arm and the latency to escape (ES) was recorded. In the retention test, the AV and ES latencies were recorded $24 \mathrm{hr}$ after without the administration of the drug. Significant impairment was observed for avoidance latencies in acquisition and retention in the SC groups compared with saline; no changes were observed for escape latencies. Similar effects but in a lesser extent were observed for diazepam in acquisition and retention avoidance latencies; an increase in escape latencies was observed suggesting an anxiolytic effect. Buspirone had no effect in acquisition and retention avoidance latencies; in the animals treated with the higher dose of BU an increase in escape latencies was observed in the first day suggesting an anxiolytic effect. Present results suggest that SC has
\end{abstract}


amnesic effects, but no anxiolytics, DZ and BU induced an anxiolytic effect, but only diazepam induced anterograde amnesia, and that these effects could be dissociated using the ETM.

Key words: memory, anxiety, elevated T maze, scopolamine, diazepam, buspirone.

Para el estudio de la memoria se han desarrollado diversos procedimientos conductuales como la prevención pasiva, el laberinto acuático de Morris, laberinto radial, etc. Con ayuda de estos procedimientos y otras manipulaciones experimentales, así como evidencia clínica en pacientes con diversas patologías de la memoria, se ha clasificado la memoria en función del tipo de información que se almacena en: memoria declarativa o explícita que a su vez se divide en memoria semántica (conocimiento del mundo) y episódica (hechos) y memoria de procedimiento o implícita que está relacionada con el aprendizaje y memoria de hábitos (habituación, condicionamiento clásico, aprendizaje no asociativo) (Squire, 2004). Numerosas evidencias indican que cada uno de estos tipos de memoria está relacionado con diferentes sistemas por lo que se ha propuesto la existencia de sistemas múltiples de memoria que tienen un sustrato neural particular (McDonald \& White, 2002). Asimismo, se ha mostrado que en los procesos de memoria participan diversos sistemas de neurotransmisión como acetilcolina, dopamina, serotonina, glutamato, entre otros, que aparentemente participan en la modulación de diferentes aspectos asociados con el aprendizaje y la memoria, como son la percepción, motivación, atención, etc. (Myher, 2003). En algunos padecimientos como la enfermedad de Alzheimer (Sims et al., 1983) y de Parkinson (Cummings, 1988), se han observado alteraciones en la concentración de diversos neurotransmisores, especialmente del sistema colinérgico.

Entre los primeros estudios en que se trató de explicar los mecanismos neurales implicados en la memoria se encuentran los de Deutch (1983) quien propuso que era importante un nivel óptimo de las concentraciones de acetilcolina en las sinapsis para el funcionamiento normal de la memoria. Para el estudio de la participación de la acetilcolina se ha empleado la administración de escopolamina (antagonista de la acetilcolina) como modelo farmacológico para inducir amnesia (Flood \& Cherkin, 1986). En el estudio de conductas motivadas aversivamente, el bloqueo de los receptores colinérgicos con escopolamina produce amnesia, efecto que depende de la magnitud del reforzamiento (Cruz-Morales, Durán-Arévalo, Díaz de Guante, Quirarte, \& Prado-Alcalá, 1992; Cruz-Morales, Reyes-Cervantes, Gómez-Romero, López \& Secundino, 1999; Quirate, Cruz-Morales, Díaz Del Guante \& Prado-Alcalá. 1993). A partir de estos resultados se ha sugerido que otros sistemas de neurotransmisión participan en la modulación de la memoria. Debido a la 
naturaleza aversiva de los estímulos empleados en el entrenamiento, uno de los candidatos es el ácido gamma amino butírico (GABA). Así, el bloqueo del sistema GABAérgico por la administración de picrotoxina y bicuculina, antagonistas con afinidad a los receptores GABAa, produce facilitación de la memoria (Brioni, 1993) y revierte la amnesia inducida por escopolamina (Cruz-Morales, 1992).

Las benzodiazepinas, en particular el diazepam, son fármacos usados comúnmente en el tratamiento de la ansiedad y se conoce que las benzodiazepinas ejercen su efecto ansiolítico interactuando con el receptor GABAa (Enna \& Bowery, 1997). El seguimiento de los pacientes tratados terapéuticamente con benzodiazepinas muestran amnesia anterógrada (Curran, 1991; Woods \& Winger, 1995), lo que indicaría un traslape en los efectos inducidos por las benzodiazepinas en los procesos de memoria y ansiedad. Estudios en animales han mostrado que las benzodiazepinas producen amnesia (Arolfo \& Brioni, 1991; Conde, Costa \& Tomaz, 1999), mientras que otros estudios muestran que la buspirona, agonista parcial de la serotonina, tiene efectos ansiolíticos y produce poco efecto en la memoria (Unregneervoort, Vanluijtrlaar, \& Coenen, 1992). Debido al amplio uso de las benzodiazepinas en el tratamiento de la ansiedad y a sus efectos secundarios (amnesia, sedación, incoordinación motora, sedación, etc.) es importante desarrollar modelos que permitan evaluar simultáneamente estos efectos.

Recientemente se desarrolló el laberinto elevado en T (LET), un procedimiento que permite evaluar en la misma prueba los efectos de los fármacos sobre la ansiedad y la memoria, (Graeff, Viana \& Tomaz, 1993; Viana, Tomaz \& Graeff, 1994). Este procedimiento deriva del laberinto elevado en X (Handley \& Mithani, 1984) o laberinto elevado en cruz (Pellow, Chopin, File \& Briley, 1985; Lister, 1987), el cual se basa en el miedo incondicionado que presentan los roedores a la altura y los espacios abiertos. Algunas de las ventajas de este procedimiento es que se mide la conducta exploratoria por periodos cortos (cinco minutos), no se requiere de privar a los animales de alimento o agua, ni la administración de estímulos aversivos como choques eléctricos.

El laberinto elevado en $\mathrm{T}$, tiene dos brazos abiertos y un brazo cerrado dispuestos en forma de T; los brazos cerrados tienen paredes de $40 \mathrm{~cm}$ de alto y el piso de los brazos se encuentra $50 \mathrm{~cm}$ arriba del piso del cuarto experimental. Los sujetos son entrenados en una tarea de evitación inhibitoria donde se registra el tiempo que tardan en salir del brazo cerrado a los brazos abiertos (latencia de evitación) y el tiempo que tardan en abandonar el brazo abierto e ingresar al brazo cerrado (latencia de escape) (Graeff et al., 1993; Viana, et al., 1994). El primer día o sesión de entrenamiento los sujetos reciben tres ensayos de evitación (los sujetos se colocan en el brazo cerrado y se mide la latencia para evitar el brazo abierto), y un ensayo de escape (los 
sujetos se colocan en el extremo de uno de los brazos abiertos y se registra la latencia para escapar de este e ingresar al brazo cerrado). Cada ensayo se realiza con un intervalo entre ensayos de $30 \mathrm{~s}$. En el segundo día se evalúa la retención, donde los sujetos reciben un ensayo de evitación y uno de escape. Se registra la actividad exploratoria y se observa que los sujetos muestran miedo incondicionado a los brazos abiertos, estas latencias para evitar los brazos abiertos son consideradas un índice de ansiedad.

El objetivo de este estudio fue evaluar en el LET los efectos del pre-tratamiento con escopolamina, un agente amnésico, ansiolíticos cuyos mecanismos de son acción diferentes, el diazepam y la buspirona. Adicionalmente se evaluó el efecto del diazepam y buspirona sobre la actividad motora.

\section{MÉTODO}

\section{Sujetos}

Los sujetos (Ss) usados fueron ratas macho Wistar ingenuos experimentalmente con un peso de 250 a $350 \mathrm{~g}$ al inicio del experimento. Los Ss se alojaron en jaulas individuales de metal con agua y alimento disponibles libremente desde tres días antes del entrenamiento, y durante todo el transcurso del estudio; los Ss fueron asignados aleatoriamente a grupos de 9 sujetos en el Experimento 2 y 3 de 10 en los Experimentos 1 y 4 .

Drogas

Todas la drogas, diazepam (Roche), escopolamina y buspirona (Sigma), fueron disueltas en solución salina isotónica $(0.9 \%)$ y administradas intraperitonealmente en un volumen de $0.1 \mathrm{ml} / 100 \mathrm{~g} / \mathrm{kg}$ de peso. El diazepam y la buspirona fueron administradas 30 minutos antes de la sesión de adquisición y la escopolamina 15 minutos antes de la sesión.

\section{Aparatos}

Actividad motora. Se empleó una cámara construida con acrílico negro de 60 $\mathrm{cm} \times 60 \mathrm{~cm} \times 60 \mathrm{~cm}$ ), provista de dos hileras de fotoceldas que permite medir la actividad horizontal (deambulación) y la actividad vertical (alzadas).

Laberinto elevado en T. El laberinto en "T" está construido en acrílico de color blanco, elevado a $50 \mathrm{~cm}$ del piso, y consiste de tres brazos de iguales dimensiones, $50 \mathrm{~cm}$ de largo y $10 \mathrm{~cm}$ de ancho, dos brazos abiertos y uno cerrado con paredes de $40 \mathrm{~cm}$ de alto, dispuestos en forma de T.

Entrenamiento: La sesión de entrenamiento consistió de tres ensayos de 
evitación y uno de escape con un intervalo de 30 s entre cada uno de ellos. En los ensayos de evitación cada $\mathrm{S}$ se colocaba en el fondo del brazo cerrado del LT y se registró la latencia para evitar los brazos abiertos, la cual se consideró como línea base (LB), seguida de dos ensayos subsecuentes de evitación (EV1, EV2). Treinta segundos después de los ensayos de evitación al $\mathrm{S}$ se le colocó al final de uno de los brazos abiertos y se registró el tiempo en que lo abandonaban e ingresaban al brazo cerrado (escape 1, ES1).

Prueba: Veinticuatro horas después de la sesión de entrenamiento (sin administrar las drogas) se realizó la prueba de retención con un ensayo de evitación y uno de escape y se registraron cada una de las latencias (EV3, ES2).

Actividad motora. Adicionalmente se realizó un experimento para evaluar actividad motora. Previamente reportamos el efecto de la administración de escopolamina sobre la actividad motora (Cruz-Morales, Reyes-Cervantes, Gomez-Romero, López- y Secundino, 1999), por lo que solo se evaluó el efecto de la administración de buspirona y diazepam sobre la actividad motora.

\section{Análisis de resultados}

Se realizaron ANOVAS de dos vías (tratamiento y ensayo) independientes para cada una de las latencias de evitación y escape, cuando se detectaron diferencias significativas se empleó la prueba de Tukey.

\section{EXPERIMENTO 1. EFECTOS DE LA ADMINISTRACIÓN DE ESCOPOLAMINA SOBRE EL LET}

En los últimos años se ha empezado a usar el laberinto elevado en T que es un procedimiento que permite evaluar tanto la memoria y la ansiedad, sin embargo existen pocos reportes donde se hayan evaluado estos dos procesos simultáneamente. La administración del anticolinérgico escopolamina ha sido utilizada como un modelo amnésico. En este estudio se empleó el LET para evaluar la memoria y la ansiedad.

\section{Procedimiento}

Los sujetos fueron asignados a 4 grupos independientes donde recibieron uno de los siguientes tratamientos de $E$ en dosis de $0.5,1$ o $3 \mathrm{mg} / \mathrm{kg}$ (E0.5, E1, E3) y otro grupo recibió solución salina en un volumen equivalente. Todas las administraciones se realizaron intraperitonealmente 15 min antes de la sesión de entrenamiento. 


\section{Resultados}

La administración de escopolamina no modificó significativamente las latencias de la línea base ni de la primera evitación. En cambio, como se muestra en la Figura $1 \mathrm{~A}$, las latencias de evitación 2 y 3 fueron significativamente menores en los Ss inyectados con las dosis altas de escopolamina que las observadas en los animales tratados con solución salina. Los análisis revelaron diferencias en las latencias de Evitación 2 en el día del entrenamiento $\left(F_{(3,32)}=8.20, p<0.001\right)$ y en las de Evitación 3 correspondientes al día de la prueba $\left(F_{(3,32)}=26.65, p<0.001\right)$.

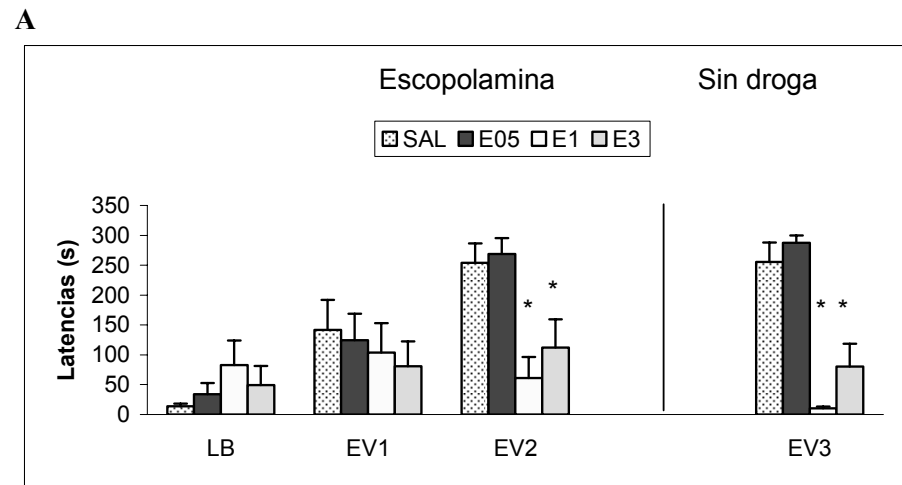

B

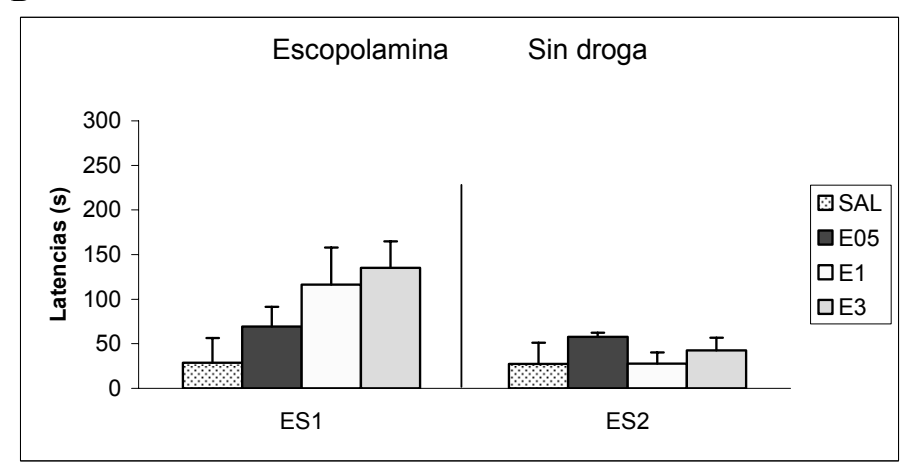

Figura 1 A) Efectos de escopolamina (E) en las latencias de evitación. *0.05 vs. salina (SAL) en el mismo ensayo; B) 2 Efectos de escopolamina (E) en las latencias de escape. 
El análisis posterior de las latencias de la Evitación 2 muestra que los grupos tratados con 1 o $3 \mathrm{mg} / \mathrm{kg}$ de escopolamina tuvieron menores latencias que las de los sujetos que recibieron salina o $0.05 \mathrm{mg} / \mathrm{kg}$ de escopolamina ( $p^{\prime} s<0.05$ ), por lo que sugerimos que la adquisición se vio afectada por la administración de escopolamina. Cuando se analizan los resultados obtenidos en el día de la prueba (evitación 3) se observa las latencias de evitación en los grupos tratados con 1 o $3 \mathrm{mg} / \mathrm{kg}$ de escopolamina fueron significativamente menores ( $p$ 's <0.001) que las de los grupos tratados con salina o 0.05 $\mathrm{mg} / \mathrm{kg}$ de escopolamina lo que indica el desarrollo de amnesia anterógrada. En las latencias de escape sólo se detectaron diferencias para el factor ensayo $\left(F_{(1,32)}=9.53, p<0.01\right)$ y no hubo efectos significativos por los tratamiento (Figura $1 \mathrm{~B}$ ).

\section{EXPERIMENTO 2. EFECTOS DE LA ADMINISTRACIÓN DE DIAZEPAM SOBRE LA EJECUCIÓN EN EL LET}

El objetivo de este experimento fue evaluar si la administración de diazepam producía cambios en memoria y en la respuesta de ansiedad en el LET.

\section{Procedimiento}

Los sujetos fueron asignados a 3 grupos independientes donde recibieron uno de los siguientes tratamientos 2 o $5 \mathrm{mg} / \mathrm{kg}$ de diazepam (DZ2, DZ5 respectivamente), y otro grupo recibió solución salina en un volumen equivalente. Las administraciones de los tratamientos se realizaron intraperitonealmente 30 min antes de la sesión de entrenamiento.

\section{RESULTADOS}

La inyección de diazepam no modificó las latencias de la línea base. La latencia de evitación en los Ss inyectados con diazepam (2 mg/kg) fue significativamente menor que en los animales inyectados con salina o $5 \mathrm{mg} / \mathrm{kg}$ de la droga. Se encontraron diferencias significativas en las latencias de evitación a lo largo de los ensayos en la sesión de adquisición para el factor droga ( $F$ $(2,24)=15.27, p<0.0001)$, el factor ensayo $\left(F_{(3,72)}=10.07\right.$, $\left.p<0.0001\right)$ y la interacción (droga $x$ ensayo) $\left(F_{(6,72)}=3.02, p<0.01\right)$. No se detectaron diferencias entre grupos en la Línea Base y Evitación 1, se detectaron diferencias en la Evitación 2 donde el grupo DZ2 difirió de los grupos de SAL y DZ5; en la Evitación 3, las latencias fueron significativamente menores en los Ss tratados con diazepam que en los inyectados con salina $\left(F_{(2,24)}=5.85\right.$, p<0.01), el 
grupo de salina difirió de los dos grupos tratados con diazepam ( $\left.p^{\prime} s<0.05\right)$. Como puede verse en la Figura $2 \mathrm{~A}$, las latencias de evitación en el día de prueba son menores en los grupos tratados con diazepam comparados con el grupo de salina lo cual sugiere que los sujetos presentaron amnesia anterógrada (Figura $2 \mathrm{~A}$ ). En las latencias de escape se encontraron efectos significativos para los factores droga $\left(F_{(2,24)}=17.21, p<0.0001\right)$, ensayo $\left(F_{(1,24)}=\right.$ $11.61, p<0.05)$ y la interacción $\left(F_{(2,24)}=3.59, p<0.05\right)$. Las latencias de escape 1 y 2 de los animales tratados $5 \mathrm{mg} / \mathrm{kg}$ de diazepam fue significativamente mayor que las de los animales inyectados con salina ( $p<0.001$; Figura 2 B).
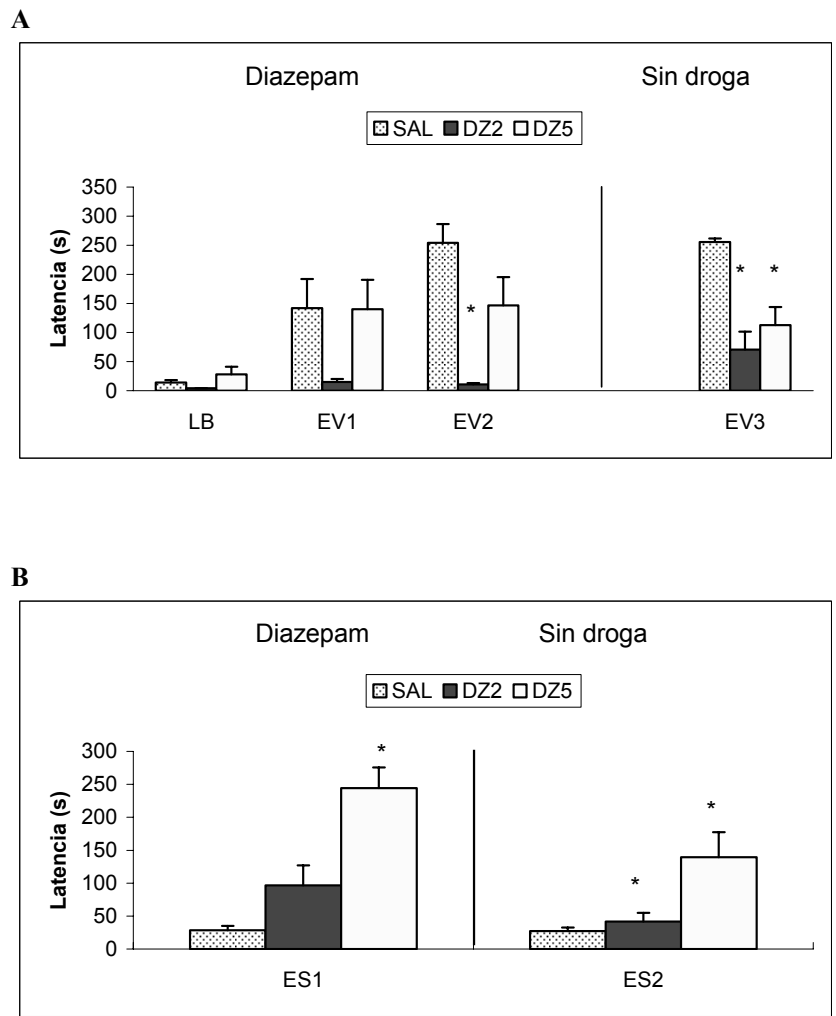

Figura 2 A) Efectos de diazepam (DZ) en las latencias de evitación. *0.05 vs. salina (SAL) en el mismo ensayo; B) Efectos de diazepam (DZ) en las latencias de escape. ${ }^{*} 0.05$ vs. salina (SAL) en el mismo ensayo. 


\section{EXPERIMENTO 3. EFECTOS DE LA ADMINISTRACIÓN DE BUSPIRONA SOBRE LA EJECUCIÓN EN EL LET}

Debido a que las benzodiazepinas además del efecto ansiolítico inducen un efecto amnésico, se han buscado drogas que puedan ser útiles en el tratamiento de la ansiedad pero sin los efectos amnésicos indeseables. La buspirona es un agonista parcial de la serotonina, se ha usado clínicamente en el tratamiento de la ansiedad por tener menos efectos amnésicos. En este estudio se evaluó el efecto de la buspirona sobre la ejecución el LET.

\section{Procedimiento}

Los sujetos fueron asignados a 3 grupos independientes donde recibieron uno de los siguientes tratamientos 2 o $5 \mathrm{mg} / \mathrm{kg}$ de buspirona (BU2, BU5 respectivamente), y un grupo recibió solución salina en un volumen equivalente. Todas las administraciones se realizaron intraperitonealmente 30 min antes de la sesión de entrenamiento.

\section{Resultados}

No se detectaron diferencias entre los grupos en ninguna de las latencias de evitación. Como puede verse en la Figura $3 \mathrm{~A}$, se observa que hay un aumento en las latencias de evitación a medida que transcurren los ensayos, lo cual es un índice de la adquisición; sin embargo no se detectaron diferencias significativas por los tratamientos en ninguna de las latencias de evitación, ni en el entrenamiento ni en la prueba. En las latencias de escape se detectaron diferencias significativas $\left(F_{(2,24)}=4,54 ; p<0.05\right)$. La latencia del escape 1 fue significativamente mayor en el grupo tratado con $5 \mathrm{mg} / \mathrm{kg}$ de buspirona que en los Ss inyectados con salina o con $2 \mathrm{mg} / \mathrm{kg}$ de buspirona $(\mathrm{p}<0.05)$. Como en el caso del diazepam se observan latencias mayores en el grupo que recibió $5 \mathrm{mg} / \mathrm{kg}$ de buspirona en el primer día, lo cual sugiere un efecto ansiolítico y no se observan cambios en las latencias de escape del segundo día (Figura $3 \mathrm{~B})$. La buspirona no afectó la adquisición ni la memoria, y produjo un efecto ansiolítico.

\section{EXPERIMENTO 4. EFECTOS DE LA BUSPIRONA Y DIAZEPAM SOBRE LA ACTIVIDAD MOTORA}

\section{Procedimiento}

Los sujetos fueron asignados aleatoriamente a grupos independientes, en el 

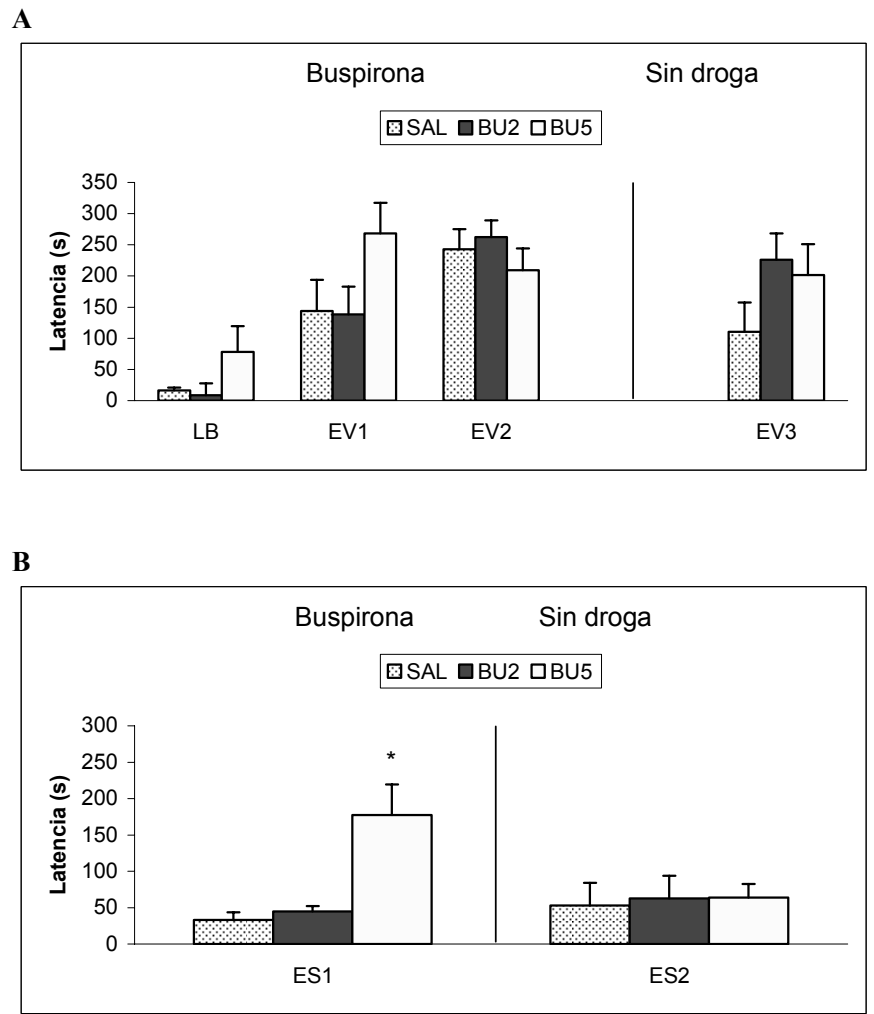

Figura 3 A) Efectos de buspirona (BU) en las latencias de evitación. *0.05 vs. salina (SAL) en el mismo ensayo; B) Efectos de buspirona (BU) en las latencias de escape. ${ }^{*} p<.05$, vs. salina (SAL) en el mismo ensayo.

caso de diazepam se emplearon tres grupos: salina, diazepam 2 y $5 \mathrm{mg} / \mathrm{kg}$; en el caso de la buspirona se empleó el mismo diseño, salina, buspirona 2 y $5 \mathrm{mg} / \mathrm{kg}$. Los fármacos se administraron y $30 \mathrm{~min}$ después los sujetos se introdujeron a la cámara donde se registró la actividad motora por $30 \mathrm{~min}$ a intervalos de 5 min.

\section{Resultados}

Se realizaron ANOVAS de medidas repetidas para cada droga y para cada tipo de actividad. En los grupos tratados con diazepam en la actividad motora horizontal sólo se observaron diferencias estadísticamente significativas para 
el factor droga $\left.F_{(2,27)}=5.27 ; p<0.001\right)$ y la interacción $\left(F_{(10,135)}=2.60 ; p<0.01\right)$; se encontró que el grupo de salina difiere del grupo DZ5 $(p<0.05)$. En la actividad motora vertical también se observó una disminución que fue estadísticamente significativa para el factor droga $\left(F_{(2,27)}=31.01 ; p<0.001\right)$, tiempo $\left(F_{(5,135)}=13.48 ; p<0.01\right)$; y la interacción $\left(F_{(10,135)}=2.60 ; p<0.01\right)$; el grupo de salina difirió de los grupos tratados con ambas dosis de diazepam $(p<0.001$ y 0.0001, respectivamente) (ver Figura 4 A y B).

A

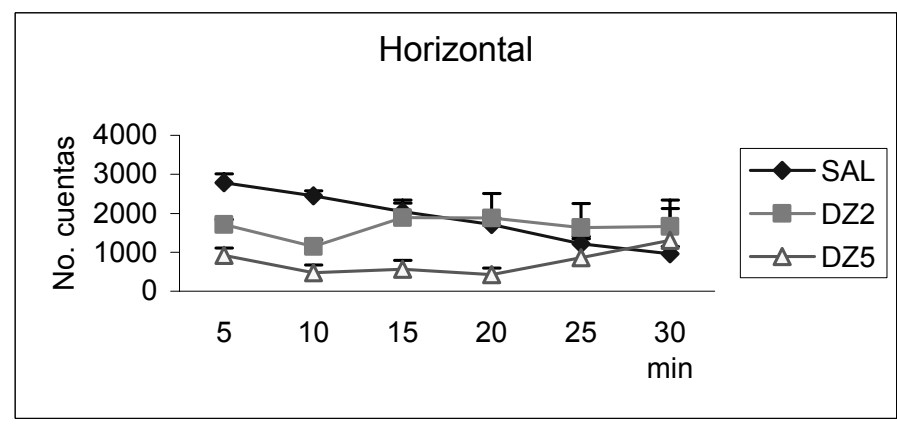

B

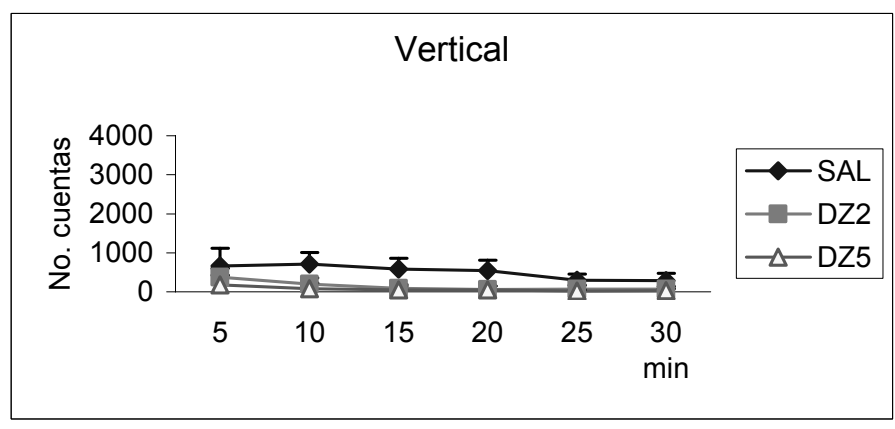

Figura 4 A) Efectos de la administración de diazepam sobre la actividad motora horizontal y vertical $(B)$.

Al igual que el diazepam, la administración de buspirona produjo una disminución de la actividad horizontal y vertical comparada con la actividad del grupo de salina (Figura $5 \mathrm{~A}$ y B). En la actividad horizontal de detectaron diferencias para el factor droga $\left(F_{(2,27)}=10.91 ; p<0.001\right)$, tiempo $\left(F_{(5,135)}=4.81\right.$; $p<0.001)$ y la interacción $\left(F_{(10,135)}=4.06 ; p<0.001\right)$. La actividad horizontal fue 
más baja en los grupos tratados con 2 y $5 \mathrm{mg} / \mathrm{kg}$ de buspirona comparados con el grupo control tratado con salina ( $p<0.001$ y 0.01 respectivamente). La actividad motora vertical también disminuyó, se encontraron diferencias para el factor droga $\left(F_{(2,27)}=90.75 ; p<0.0001\right)$, tiempo $\left(F_{(5,135)}=12.31 ; p<0.0011\right)$ y la interacción $\left(F_{(10,135)}=9.47 ; p<0.0001\right)$, los dos grupos que recibieron buspirona difirieron del grupo salina $(p<0.0001)$.

A

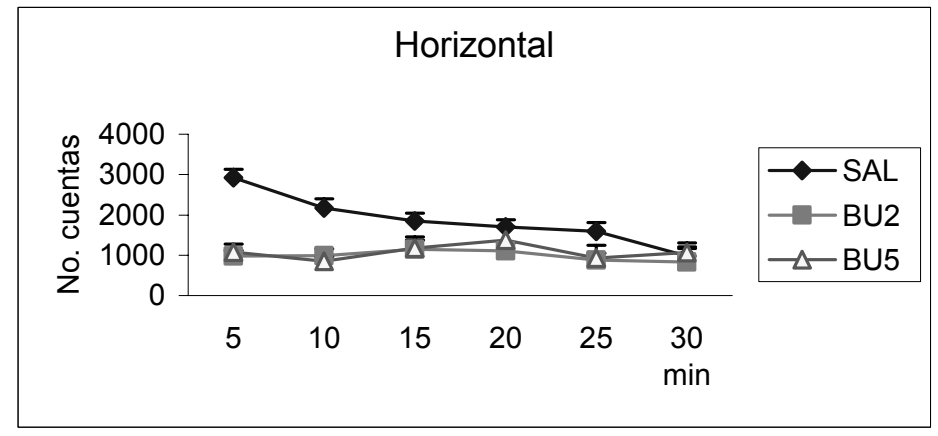

B

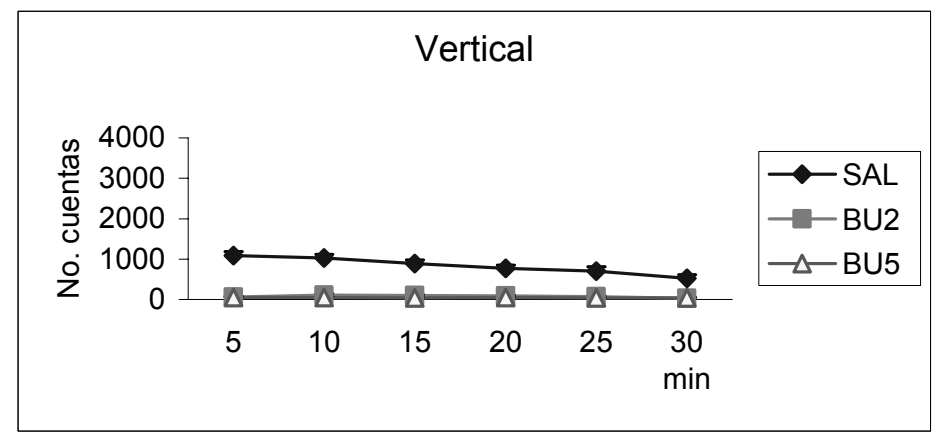

Figura 5 A) Efectos de la administración de buspirona sobre la actividad motora horizontal y vertical $(B)$.

\section{Discusion}

El laberinto elevado en Tha sido validado para medir ansiedad y memoria (Viana, et al., 1994; Zangrossi \& Graeff, 1997). Los resultados del presente estudio demuestran que en los grupos tratados con solución salina las latencias de 
evitación aumentan a lo largo de los ensayos lo cual es un índice de una buena adquisición y una buena retención cuando son evaluados a las veinticuatro horas, ya que los sujetos evitan por más tiempo los brazos abiertos.

Las diferencias detectadas por tratamiento indican diferencias entre los grupos tratados con escopolamina y el grupo de salina en las latencias de evitación. Como puede verse en la Figura 1 A las latencias del grupo tratado con salina aumentaron con el número de ensayos, lo cual demuestra que los sujetos mostraron una buena adquisición, mientras que en los grupos que recibieron escopolamina, aunque también hay un aumento en las latencias de evitación, éstas son mucho menores, particularmente en los grupos tratados con 1 y $3 \mathrm{mg} / \mathrm{kg}$ de escopolamina; estos datos sugieren que los grupos tratados con escopolamina muestran deterioro en la adquisición. En la prueba de retención estos mismos grupos (E1 y E3) difirieron del grupo de SAL lo cual demuestra que la escopolamina indujo amnesia anterógrada, no se observaron diferencias con la menor dosis de escopolamina. Estos datos coinciden con otros reportes en ratas donde se han empleado pruebas diferentes como evitación inhibitoria (Cruz-Morales, et al., 1999; Elrod \& Buccafusco, 1988), laberinto acuático (De-Mello \& Carobrez, 2002; Stone, Croul \& Gold, 1988) en ratas y en ratones (Rush, 1988). Recientemente se ha propuesto que el LET puede ser usado como un modelo para medir memoria y que sus efectos no se deben a los efectos sobre ansiedad (De-Mello \& Carobrez, 2002). En ese estudio se usó un protocolo diferente de entrenamiento que consistió en entrenar a los sujetos hasta que alcanzaran el criterio de evitación de permanecer $300 \mathrm{~s}$ en el brazo cerrado y 72 horas después fueron evaluados. No se detectaron efectos con dosis de 0.3 de escopolamina en la adquisición, medida por el número de ensayos para alcanzar el criterio, mientras que el grupo tratado con $1.2 \mathrm{mg} / \mathrm{kg}$ de escopolamina se observó un deterioro en la adquisición comparado con su grupo control de salina. Se reportó deterioro en la retención medida a las $72 \mathrm{~h}$ con ambas dosis.

En la prueba de LET la latencia de escape es interpretada usualmente como índice de ansiedad, latencias largas indican un efecto ansiolítico y latencias menores denotan mayor grado de ansiedad. En el estudio de DeMello y Carobrez (2002) no se midió el escape en el LET, pero se empleó la prueba del laberinto elevado en cruz y se detectaron cambios en dos respuestas que son empleadas como índices de ansiedad (número de entradas a brazos abiertos y disminución de riesgo) lo cual parecería indicar un efecto ansiolítico, sin embargo también se detectaron cambios en el número de entradas a los brazos abiertos lo cual sugiere que los efectos de la escopolamina podrían atribuirse más bien a cambios en la actividad motora.

Rodgers y Cole (1995) en ratones evaluados en el laberinto elevado en cruz, describió el efecto ansiolítico de escopolamina con dosis de $1 \mathrm{mg} / \mathrm{kg}$ inferido por el aumento de entradas a los brazos cerrados y algunas respues- 
tas etológicas, aunque no se reportaron efectos significativos en el número de entradas a los brazos abiertos o en el porcentaje de entradas a brazos abiertos. Parte de las diferencias pueden deberse a que se empleó un procedimiento diferente y que el autor sugiere que la escopolamina produjo un incremento en la actividad motora.

En los resultados del presente estudio no se detectaron diferencias estadísticamente significativas en las latencias de escape debidas al tratamiento, lo cual nos lleva a pensar que en estas condiciones la escopolamina no tiene efectos ansiolíticos. Sin embargo es interesante notar que en la latencia del primer escape se observa, comparando con el grupo de salina, un aumento dependiente de la dosis de escopolamina, lo cual podría sugerir un efecto ansiolítico. Esto se podría explicar otra vez por los cambios inducidos por la escopolamina en la actividad motora como se ha demostrado previamente, donde dosis equivalentes producen una disminución de la actividad, lo cual podría explicar el aumento de la latencia de escape (Cruz-Morales, et al., 1999). En resumen, en los grupos tratados con escopolamina se observaron latencias menores tanto en la adquisición como en la retención comparada con los grupos de salina y no se observaron diferencias en las latencias de escape. Por tanto la administración pre-entrenamiento de escopolamina produjo deterioro en la adquisición, indujo amnesia anterógrada pero no tuvo efectos ansiolíticos ya que no produjo efectos significativos en las latencias de escape.

En el caso del diazepam, como puede verse en la Figura $2 \mathrm{~A}$, durante el entrenamiento las latencias de evitación aumentan a través de los ensayos lo cual indica que hay una buena adquisición especialmente en el grupo tratado con salina, mientras que se observan menores latencias en los grupos tratados con diazepam lo cual sugiere que el diazepam induce un deterioro en la adquisición, lo que coincide con reportes previos en el laberinto elevado en T con ratas como sujetos (Graeff, Ferreira-Netto \& Zangrossi, 1998; Viana et al., 1994) y con ratones (Carvalho-Netto \& Nunes-de-Souza, 2004). Sin embargo no coincide con trabajos previos donde no se observan efectos del diazepam sobre la adquisición en el laberinto elevado en T (Conde et al., 1999; Echeverry, Hasenöhrl, Huston \& Tomaz, 2001) y en evitación inhibitoria (Gómez-Romero, Reyes, Arriaga \& Cruz-Morales, 2002) y se demuestra una disociación entre los efectos del diazepam sobre la retención pero no sobre la adquisición (Cole \& Jones, 1995). En algunos de los casos la diferencia se puede explicar por las diferencias en las tareas. En el caso de (Cole \& Jones, 1995 y en el de Gómez-Romero et al., 2002), se empleó la tarea de evitación inhibitoria en una cámara de dos compartimientos, en este procedimiento se administran choques y usualmente es suficiente con un ensayo para adquirir la respuesta. Lo anterior nos hablaría de que la administración del choque es una situación más aversiva que los espacios abiertos del LET y por tanto puede ser menos sensible a cambios en la adquisición. Por otro 
lado, existen diferencias en el número de ensayos; como ya se mencionó en evitación inhibitoria se emplea un ensayo y en el LET tres ensayos, la importancia del número de ensayos queda de manifiesto cuando en el LET se emplean un mayor número de ensayos hasta alcanzar el criterio, entonces la adquisición no se ve afectada (Conde et al., 1999). Lo anterior coincide con otros reportes donde la amnesia parece depender del grado de entrenamiento (Prado-Alcalá \& Cobos-Zapiaín, 1977) y de la intensidad del estímulo aversivo (Cruz-Morales, et al., 1992). En las latencias de retención o EV3 evaluadas veinticuatro horas después en ausencia de la droga, nuevamente se observa un deterioro en los grupos tratados con diazepam, comparados con el grupo de salina lo cual muestra que el pre-tratamiento con diazepam induce amnesia anterógrada, lo que previamente reportamos empleando un procedimiento de evitación inhibitoria (Gómez-Romero et al., 2002). Lo anterior sugiere que el efecto del diazepam es tal porque incide sobre el proceso de consolidación y sobre la adquisición, lo cual es consistente con datos en humanos donde el diazepam deterioró la memoria (Lucki, Rickels, Giesecke \& Geller, 1987).

Por otro lado, en el análisis del las latencias de escape se observa un aumento importante en las latencias de escape el primer día, lo cual sugiere el efecto ansiolítico del diazepam, y un incremento menor en el segundo día. Es interesante notar que estos efectos no coinciden con reportes previos donde el efecto ansiolítico no se detecta en las latencias de escape (Conde et al, 1994; Graeff et al., 1993; Graeff, et. al, 1998; Viana et al., 1994). Sin embargo coincide ampliamente con el trabajo de Echeverry et al. (2002) donde el diazepam aumentó las latencias de escape en forma importante el primer día, sugiriendo un efecto ansiolítico, y en menor grado el segundo día, sugiriendo un efecto sobre la memoria.

Es importante señalar que existen inconsistencias con respecto a los efectos de la buspirona, tanto sobre la memoria (Curran, 1991) como en las pruebas diseñadas para evaluar la ansiedad (Cole \& Rodgers, 1994). Por ejemplo, en ratones, se reportó un deterioro en la adquisición de la respuesta de evitación en el LET con dosis de 1 y $2 \mathrm{mg} / \mathrm{kg}$ con un procedimiento de cinco ensayos de evitación, mientras que en el escape se encontró una disminución de la latencia, lo cual fue interpretado como facilitación de la respuesta (Carvalho-Netto et al., 2004). En ratas evaluadas en el LET usando el mismo procedimiento que en el presente trabajo, la buspirona $(0.3,1,3 \mathrm{mg} /$ $\mathrm{kg}$ ) produjo un deterioro en la respuesta de evitación con $0.3 \mathrm{mg} / \mathrm{kg}$ y no se detectaron cambios en el Escape 1 (Graeff et al., 1998); efectos semejantes se han reportado en evitación pasiva, evitación activa y laberinto de Morris (Liang, Tsui, Tyan \& Chiang, 1998). A diferencia de estos reportes se ha demostrado que la pre-administración de buspirona en ratas facilita o no tiene efectos en la adquisición de una respuesta de evitación pasiva y deteriora la 
retención cuando es administrada después del entrenamiento (Zhang, Yuan \& Luo, 2000). En el presente estudio la administración de buspirona no tuvo efectos importantes sobre la adquisición de la respuesta de evitación el día del entrenamiento, ni en la memoria ya que no tuvo efectos en la latencia de retención el día de la prueba. Efectos similares para la respuesta de evitación en ratas fueron reportados previamente en la prueba de evitación inhibitoria en una cámara de dos compartimientos donde se reportó amnesia anterógrada para diazepam pero no para buspirona y que este efecto no fue dependiente del estado (Gómez-Romero, et al, 2002). Estos datos coinciden con lo reportado donde la buspirona no produce daño en la memoria en humanos (Lucki, et al., 1987) y en ratas donde la administración de buspirona antes del entrenamiento en evitación pasiva no produce efectos sobre la adquisición (Zhang, et al, 2000). En nuestros resultados encontramos un aumento en la latencia de escape el primer día que interpretamos como un efecto ansiolítico de la buspirona lo cual concuerda con los efectos ansiolíticos reportados en el laberinto elevado en cruz (Cole \& Rodgers, 1994). Por lo anterior es importante considerar que diferencias en la tarea que se emplea para medir ansiedad y memoria, del procedimiento, tiempo de administración, dosis, etc., son variables que pueden explicar algunas de estas inconsistencias.

Es importante discutir los efectos sobre actividad motora, el diazepam y la buspirona produjeron disminución en la actividad motora. Tanto en la actividad horizontal como en la vertical se detectaron diferencias entre el grupo tratado con solución salina y los grupos tratados con buspirona en ambas dosis. Esto es consistente con lo reportado en ratas donde la buspirona (1.0, $4.0 \mathrm{mg} / \mathrm{kg}$ ) produjo una disminución en la actividad motora medida como el número de entradas a brazos abiertos y cerrados y la distancia recorrida en los brazos en el laberinto elevado en cruz (Collinson \& Dawson, 1997). Sin embargo con dosis menores de 1 y $2 \mathrm{mg} / \mathrm{kg}$ de diazepam y buspirona, no se han detectado cambios en la actividad motora en ratones (Carvalho-Netto et al., 2004).

Cuando la administración de drogas altera la actividad motora, en el LET la alteran en el mismo sentido tanto en la evitación como en el escape, en esos casos son necesarias pruebas que permitan evaluar en forma específica la actividad motora. Lo interesante es que aunque hay disminución en la actividad motora con buspirona y con diazepam, los cambios no coinciden con lo observado en adquisición, retención y ansiedad. Por ejemplo, en el caso del diazepam con 2 pero no con $5 \mathrm{mg} / \mathrm{kg}$, se observó déficit en la adquisición, y se detectó daño en la retención a las $24 \mathrm{~h}$ con ambas dosis cuando los sujetos fueron evaluados sin el efecto de la droga. En otras palabras, las dosis de diazepam que diminuyeron la actividad motora, produjeron una disminución en las respuestas de evitación y un aumento en las de escape, no solo en el primer día sino también en el segundo lo cual nos habla del 
deterioro en la retención ya que a las $24 \mathrm{~h}$ los animales fueron evaluados sin la droga. Mientras que en el caso de la buspirona ambas dosis disminuyeron la actividad motora, y no disminuyeron las latencias de evitación ni el día del entrenamiento ni el día de la prueba, por lo que se puede afirmar que no tuvo efecto en la adquisición ni en la retención, a pesar de la disminución de la actividad motora. En el escape solo una dosis produjo un aumento en la latencia el día del entrenamiento.

La controversia sobre los efectos de las benzodiazepinas sobre la memoria, puede ser explicada en parte, porque las benzodiazepinas pueden actuar y afectar en forma diferencial los diferentes tipos de memoria. Datos clínicos en humanos han demostrado que la administración de benzodiazepinas afecta la memoria declarativa pero no la memoria de procedimiento. Sin embargo los presentes resultados muestran que el diazepam produjo deterioro en la adquisición y retención de la memoria de procedimiento y que los efectos no pueden ser atribuidos a efectos inespecíficos sobre la actividad motora. Por otro lado, las inconsistencias de los efectos de la buspirona nos sugieren diferencias en la sensibilidad de los procedimientos para medir ansiedad y los diferentes tipos de ansiedad, así como diferencias en los procedimientos para medir diferentes tipos de memoria. Finalmente estos resultados sugieren que el procedimiento del LET permite disociar los efectos amnésicos de los efectos ansiolíticos.

\section{REFERENCIAS BIBLIOGRÁFICAS}

Arolfo, M. P. \& Brioni, J. D. (1991). Diazepam impairs place learning in the Morris water maze. Behavioral and Neural Biology, 55, 131-136.

Brioni, J. (1993). Role of GABA during the multiple consolidation of memory. Drug Development Research, 28, 321-324.

Carvalho-Netto, E. F. \& Nunes-de-Souza, R. L. (2004). Use of the elevated T-maze to study anxiety in mice. Behavioural Brain Research, 148, 119-132.

Cole, B. J. \& Jones, G. H. (1995). Double dissociation between the effects of muscarinic antagonists and benzodiazepine receptor agonists on the acquisition and retention of passive avoidance. Psychopharmacology, 118, 37-41.

Cole, J. C. \& Rodgers, R. J. (1994). Ethological evaluation of the effects of acute and chronic buspirone treatment in the murine elevated plus-maze: comparison with haloperidol. Psychopharmacology, 114, 288-296.

Collinson N. \& Dawson, G. R. (1997). On the elevated plus-maze the anxiolytic-like effect of 5- $\mathrm{HT}_{1 \mathrm{a}}$ agonist, 8-OH-DPAT, but not the anxiogenic-like effects of the $5-\mathrm{HT}_{1 \mathrm{a}}$ partial agonist, buspirone, are blocked by the $5-\mathrm{HT}_{1 \mathrm{a}}$ antagonist, WAY 100635. Psychopharmacology, 132, 35-43.

Conde, C. A., Costa, V., \& Tomaz, C. (1999). Measuring emotional memory in the elevated T-maze using a training-to-criterion procedure. Pharmacology Biochemistry \& Behavior, 63, 63-69. 
Cruz-Morales, S. E. (1992). Interacción de los sistemas colinérgico y gabaérgico en la consolidación de una respuesta de evitación inhibitoria. Tesis Doctorado, Fac. Medicina, UNAM.

Cruz-Morales, S. E., Durán-Arévalo, M., Díaz de Guante, M. A., Quirarte, G., \& PradoAlcalá, R. (1992). A threshold for the protective effect of over-reinforced passive avoidance against scopolamine-induced amnesia. Behavioral and Neural Biology, 57, 256-259.

Cruz-Morales, S. E., Reyes-Cervantes, G., Gómez-Romero, J. G., López, Y. \& Secundino, I. (1999). La escopolamina produce amnesia retrógrada en evitación inhibitoria entrenada con diferentes magnitudes de reforzamiento. Revista Mexicana de Psicología, 16 (2), 195-202.

Cummings, J. L. (1988). The dementias of Parkinson's disease: prevalence, characteristics, neurobiology, and comparison with dementia of the Alzheimer type. European Neurology, 28, 1, 15-23.

Curran, H.V. (1991). Benzodiazepines, memory and mood: a review. Psychopharmacology $1051-8$.

Deutch, A. J. (1983). The cholinergic synapse and the site of memory. En A. J. Deutch (Ed.) The physiological basis of memory, (pp 367-386). New York: Academic Press, Inc.

De-Mello, N. \& Carobrez, A. P. (2002). Elevated T-maze as an animal model of memory: effects of scopolamine. Behavioural Pharmacology, 13, 139-148.

Echeverry, M. B., Hasenöhrl, R. U., Huston, J. P. \& Tomaz, C. (2001). Comparison of neurokinin SP with diazepam in effects of memory and fear parameters in the elevated T-maze free exploration paradigm. Peptides 22, 1031-1036.

Elrod, K. \& Buccafusco, J. J. (1988). An evaluation of mechanism of scopolamine induced impairment in two passive avoidance protocol. Pharmacology Biochemistry \& Behaviour, 29, 15-21.

Enna, S. J. Bowery, N. G. (1997). The GABA Receptors, 2nd ed. Clifton, NJ: Humana Press.

Flood, J. F. \& Cherkin, A. (1986). Scopolamine effects on memory retention in mice: A model of dementia? Behavioral and Neural Biology, 45, 169-184.

Graeff, F. G., Viana, M. B. \& Tomaz, C. (1993). The elevated T maze, a new experimental model of anxiety and memory: effect of diazepam. Brazilian Journal of Medical and Biological Research, 26, 67-70.

Graeff, F. G., Ferreira-Netto, C., \& Zangrossi, H. Jr. (1998). The elevated T-maze as an experimental model of anxiety. Neuroscience \& Biobehavioral Reviews, 23, 237-246.

Gómez-Romero, J. G., Reyes, G., Arriaga, P. \& Cruz-Morales, S. E. (2002). Efectos de drogas ansiolíticas sobre la adquisición de una tarea de evitación pasiva. En: Hernández-Pozo, R., Arriaga Ramírez, P. \& López Rodríguez (Eds.), Perspectivas de la Psicología Experimental en México, Vol. 2, (Pp. 1-11). F. UNAM, FESIztacala,

Handley, S. L. \& Mithani, S. (1984). Effects of alpha-adrenoreceptors agonist and antagonist in a maze-exploration model of "fear" motivated behaviour, NaunySchimiedeberg's Arch Pharmacol, 327, 1-5).

Liang, K.-C., Tsui, K.-Y., Tyan, Y.-M., \& Chiang, T.-C. (1998) Buspirone impaired acqui- 
sition and retention in avoidance tasks: Involvement of the hippocampus. Chinese Journal of Physiology 41 (1), 33-44.

Lister, R. G. (1987). The use of a plus-maze to measure anxiety in the mouse. Psychopharmacology, 92, 180-185.

Lucki, I., Rickels, K., Giesecke, M. A. \& Geller, A. (1987). Differential effects of the anxiolytic drugs, diazepam and buspirone, on memory function. British Journal of Clinical Pharmacology, 23, 207-211.

McDonald, R. J. \& White, N. (2002). Multiple parallel memory systems in the brain of the rat Neurobiology of Learning and Memory, 77,125-184.

Myhrer, T. (2003). Neurotransmitter systems involved in learning and memory in the rat: a meta-analysis based on studies of four behavioral tasks. Brain research Reviews, 41, 268-287.

Pellow, S., Chopin, P., File, S. E., \& Briley, M. (1985). Validation of open: closed arm entries in an elevated plus-maze as a measure of anxiety in rats. Journal of Neuroscience Methods, 14, 149-167.

Prado-Alcalá, R.A. \& Cobos-Zapiaín, G. G. (1977). Learning deficits induced by cholinergic blockade of the caudate nucleus as a function of experience. Brain $R e-$ search, 138 (1), pp. 190-196

Quirarte, L., Cruz-Morales, S. E., Díaz del Guante, M. A. \& Prado-Alcalá, R. A. (1993) Protective effect of under-reinforcement of passive avoidance against scopolamine induced amnesia. Brain Research Bulletin, 32, 521-524.

Rodgers, R. J. \& Cole, J. C. (1995). Effects of scopolamine and it quaternary analogue in the murine elevated plus-maze test of anxiety. Behavioral Pharmacology, 6, 283-289.

Rush, D. K. (1988). Scopolamine amnesia of passive avoidance: A deficit of information acquisition. Behavioral and Neural Biology, 50, 255-274.

Sims, N. R., Bowen, D. M., Alle, S. J., Smith, C. C. T., Neary, D., Thomas, D. J., \& Davidson, A. N. (1983). Presynaptic cholinergic dysfunction in patients with dementia. Journal of Neurochemistry, 40, 503-509.

Squire, L. R. (2004). Memory systems of the brain: A brief history and current prospective. Neurobiology of Learning and Memory, 82, 171-177.

Stone, W. S., Croul, C. E. \& Gold, P. E. (1988). Attenuation of scopolamine-induced amnesia in mice. Psychopharmacology 96 (3), pp. 417-420

Viana, M. B., Tomaz, C., \& Graeff, F. G. (1994). The elevated T-Maze: a new animal model of anxiety and memory. Pharmacology, Biochemistry \& Behavior, 49, 549554.

Unregneervoort, A., Vanluijtrlaar, G. \& Coenen, A. (1992). Cognition and vigilance: Differential effects of diazepam and buspirone on memory and psychomotor performance, Neuropsychobiology, 26, 146-150.

Woods, J. H. \& Winger, G. (1995). Currents benzodiazepines issues. Psychopharmacology, 118, 107-115.

Zangrossi, H. Jr. \& Graeff, F. G. (1997). Behavioral validation of the elevated T-maze, a new animal model of anxiety. Brain Research Bulletin, 44 (1), 1-5.

Zhang, Z.-Q., Yuan, L., \& Luo, Z.-P. (2000) Effects of buspirone on active and passive avoidance responses in mice. Chinese Journal of Pharmacology and Toxicology $14,461-463$. 